\title{
Cálculo da taxa de ionização do átomo de hidrogênio em campo elétrico uniforme via método WKB
}

(Semiclassical calculation of ionization rate of hydrogen in a uniform electric field)

\author{
Ariel Almeida Abreu Silva e A.V. Andrade-Neto ${ }^{1}$ \\ Departamento de Física, Universidade Estadual de Feira de Santana, Feira de Santana, BA, Brazil \\ Recebido em 16/10/2009; Aceito em 15/3/2010; Publicado em 17/1/2011
}

\begin{abstract}
Neste artigo calculamos a taxa de ionização para o átomo de hidrogênio na presença de um campo elétrico uniforme. A probabilidade de penetração da barreira é calculada usando a aproximação WKB. Para a realização desse cálculo utilizamos dois modelos de potenciais unidimensionais: no primeiro modelo o potencial coulombiano é considerado apenas na região próxima ao núcleo atômico, o que permite uma solução exata da integral a ser resolvida. No segundo modelo o potencial coulombiano é levado em conta em todo o espaço, o que leva a uma integral cuja solução deixa de ser trivial. Contudo, é possível resolvê-la em termos de integrais elípticas. Os resultados obtidos são comparados com o resultado aceito como o correto $\left[4 F^{-1} \exp (-2 / 3 F)\right]$ devido a Landau e Lifshitz.
\end{abstract}

Palavras-chave: ionização por campo, efeito túnel, método WKB.

In this paper we calculated the model dependence of the field ionization rate of hydrogen atom in a uniform electric field. In order to calculate the probability of barrier penetration we use semiclassical (WKB) approximation, which is calculated for two model potentials. In the model 1 the Coulomb potential is used only near the nucleus. In model 2 the Coulomb potential is used in all regions. These results are compared with the accepted result of $4 F^{-1} \exp (-2 / 3 F)$

Keywords: field ionization, tunnel effect, WKB approximation.

\section{Introdução}

O átomo de hidrogênio é um dos poucos sistemas mecânico quântico de interesse físico que possui solução analítica exata. Além desse fato, esse sistema é de grande importância por, no mínimo, dois motivos: historicamente ele foi o primeiro problema no qual Schrödinger aplicou a equação que leva o seu nome, determinando os autoestados e os autovalores da energia. Por outro lado, a solução para o átomo de hidrogênio pode ser adaptada para sistemas mais complexos.

Igualmente importante é o problema do átomo de hidrogênio na presença de um campo elétrico. Para um campo uniforme na direção do eixo $z$, a equação de Schrödinger independente do tempo fica (no sistema CGS)

$$
\left[-\frac{\hbar^{2}}{2 m} \nabla^{2}-\frac{e^{2}}{r}+F e z\right] \psi(\mathbf{r})=E \psi(\mathbf{r})
$$

onde $\hbar$ é a constante de Planck, $e$ e $m$ são, respectivamente, a carga e a massa do elétron, $F$ é a magnitude do campo elétrico, $E$ é a energia total da partícula e $\psi$ é a função de onda eletrônica.

Nesse trabalho utilizaremos o sistema de unidades atômicas no qual se faz $\hbar=m=e=1$. Para maiores detalhes ver o Apêndice. Nesse sistema a Eq. (1) fica

$$
\left[-\frac{\nabla^{2}}{2}-\frac{1}{r}+F z-E\right] \psi(\mathbf{r})=0
$$

onde $z=r \cos \theta$ e $\theta$ é o ângulo entre $r$ e o eixo $z$.

A aplicação do campo elétrico influencia o átomo de duas maneiras: o campo provoca um desdobramento dos níveis de energia do átomo, o que constitui o efeito Stark, e, em segundo lugar, para campos suficientemente intensos (da ordem de alguns volts/angstrons) a barreira de potencial pode ser reduzida de maneira a tornar finita a probabilidade de o átomo ser ionizado pelo tunelamento do seu elétron. No presente trabalho estamos interessados nesse último efeito. Tunelamento ou penetração de barreira é a possibilidade de uma partícula com energia total $E$ penetrar em uma região de energia potencial $V$, onde $E<V$, fato que é impossível na mecânica clássica. Contudo, há um análogo ondulatório clássico desse efeito que é a reflexão interna

\footnotetext{
${ }^{1}$ E-mail: aneto@uefs.br. 
total frustrada, um fenômeno bem conhecido da teoria eletromagnética da luz [ver, por exemplo, a seção 5.10 da Ref. [1]]. A penetração de barreira ou efeito túnel é muito comum em sistemas atômicos e moleculares e é o principio básico de vários dispositivos tecnológicos e alguns microscópios que apresentam resolução atômica.

A ionização de um átomo, por tunelamento do seu elétron, devido a presença de um campo elétrico é conhecido como ionização por campo e será objeto de análise do presente trabalho.

O primeiro a demonstrar essa possibilidade foi o físico americano Robert Oppenheimer [2] em 1928, como uma previsão teórica da então nascente teoria quântica. Ele obteve que o efeito seria apreciável para campos da ordem de $0,5 \mathrm{~V} / \AA$. Apenas na década de 1950, com a criação do microscópio iônico de campo, mais conhecido pela sigla FIM (da acrossemia em inglês Field Ion Microscope) foram obtidos campos com magnitudes suficientes para realizar a ionização por campo. O FIM foi o primeiro instrumento inventado pelo homem com capacidade de obter imagens individuais de átomos [3]. Desse modo, o fenômeno de ionização por campo próximo a uma superfície metálica tornou-se um problema de grande interesse científico.

A utilização de coordenadas parabólicas na abordagem do problema do átomo de hidrogênio na presença de um campo elétrico é amplamente utilizada já que nesse sistema a equação de Schrodinger, Eq. (1), é exatamente separável. Desde o trabalho pioneiro de Oppenheimer, esse problema tem sido o exemplo favorito para aplicação de novas teorias e métodos $[4,5]$.

Neste trabalho apresentaremos um método relativamente simples para o cálculo da taxa de ionização do átomo de hidrogênio quando sujeito a um campo elétrico externo. Utilizaremos alguns modelos unidimensionais, adaptados da Ref. [5], que representam a energia potencial de um elétron sujeito a um campo coulombiano e a um campo elétrico externo. Para calcular a probabilidade de penetração de barreira utilizamos a aproximação WKB, que é uma ferramenta matemática acessível a estudantes de graduação de um curso de Física e que pode ser utilizada na abordagem de problemas de grande interesse científico e tecnológico [6]. O método apresentado aqui possui a vantagem de permitir um tratamento analítico para processos mais complexos de ionização por campo como, por exemplo, a ionização de um gás próximo à superfície de um metal $[5,7]$.

Este trabalho está organizado da seguinte forma: na Seção 2 discorremos sobre a taxa de ionização e a expressão para o cálculo dessa grandeza é deduzida. Na Seção 3 é realizado o cálculo da probabilidade de penetração de barreira, via aproximação WKB, e apresentamos os modelos para os potenciais unidimensionais utilizados. Na Seção 4 escrevemos as expressões obtidas nesse trabalho para a taxa de ionização e comparamos com a expressão de Landau e Lifshitz. Na Seção 5 apresentamos nossas conclusões e consideraçãos finais.

\section{A taxa de ionização}

Como declarado acima, o objetivo principal do presente trabalho é calcular a taxa de ionização do átomo de hidrogênio quando submetido a um campo elétrico externo. Para isso, consideraremos o elétron preso em um poço de potencial unidimensional se chocando a uma certa taxa por unidade de tempo contra a barreira. A taxa de ionização $I$ é a probabilidade, por unidade de tempo, de que o elétron escape do átomo. Chamando de $\nu$ o número de tentativas por unidade de tempo, i.e., o número de choques, por unidade de tempo, realizado pelo elétron contra a barreira, temos a seguinte expressão para $I$

$$
I=\nu P
$$

onde $P$ é a probabilidade de que em uma tentativa o elétron escape do átomo.

A grandeza $\nu$ pode ser calculada pela expressão

$$
\nu=\frac{v}{2 a},
$$

onde $v$ é a velocidade do elétron no interior da barreira de largura 2a. Utilizaremos o modelo de Bohr para calcular a Eq. (4). Considerando o elétron no estado fundamental temos que (no sistema $\operatorname{cgs}$ ) $v=v_{o}=e^{2} / \hbar$ ( a velocidade do elétron na primeira órbita de Bohr) e $a=a_{o}=\hbar^{2} /\left(m e^{2}\right)$ ( o raio de Bohr). Logo, no sistema de unidades atômicas encontramos

$$
\nu=\frac{1}{2} .
$$

Calculado $\nu$ precisamos agora encontrar a probabilidade de penetração de barreira.

\section{Cálculo da probabilidade de pene- tração de barreira}

Para o cálculo da probabilidade de penetração da barreira de potencial, $P$, utilizaremos a aproximação WKB (devido a Wentzel, Kramers e Brillouin). Esse método, também conhecido como semiclássico, fornece a seguinte expressão $[8,9]$ para $P$

$$
P=\exp \left[-2^{3 / 2} \int_{x_{1}}^{x_{2}} \sqrt{V(x)-E} d x\right]
$$

onde $V(x)$ é a energia potencial, $E$ é a energia total da partícula e $x_{1}$ e $x_{2}$ são os pontos de retorno clássico nos quais, por definição, $V(x)=E$.

O potencial unidimensional para o átomo de hidrogênio na presença de um campo elétrico uniforme, $\mathbf{F}=-F \hat{x}$, onde $\hat{x}$ é o vetor unitário do eixo $x$, é dado por 


$$
V(x)=-\frac{1}{|x|}-F x
$$

onde o primeiro termo, $-1 /|x|$, representa a energia potencial coulombiana e o termo $-F x$ representa a energia potencial devida ao campo elétrico (Fig. 2).

A energia total do elétron será a sua energia de ligação, denominada de $B$, que é negativa, i.e., $E=-B$. Desse modo, a barreira de potencial é dada por

$$
V(x)-E=B-\frac{1}{|x|}-F x .
$$

Os pontos de retorno clássico são as raízes da Eq. (8) dadas pela expressão

$$
x=\frac{B \pm \sqrt{B^{2}-4 F}}{2 F} .
$$

Valores típicos de $F$ e $B$, em unidades atômicas, são, respectivamente, $0,01(0,514 \mathrm{~V} / \AA)$ e $0,5(13,6 \mathrm{eV})$. Desse modo, podemos considerar que $F<<B^{2}$. Utilizando essa aproximação, os pontos de retorno clássico são escritos como

e

$$
x_{1}=\frac{1}{B}
$$

$$
x_{2}=\frac{B}{F} .
$$

A barreira de potencial apresenta um máximo em (veja Fig. 1)

$$
x_{\max }=\frac{1}{F^{1 / 2}} .
$$

Para resolver a Eq. (6) vamos considerar dois modelos para o potencial, que denominaremos modelo $1 \mathrm{e}$ modelo 2 descritos a seguir.

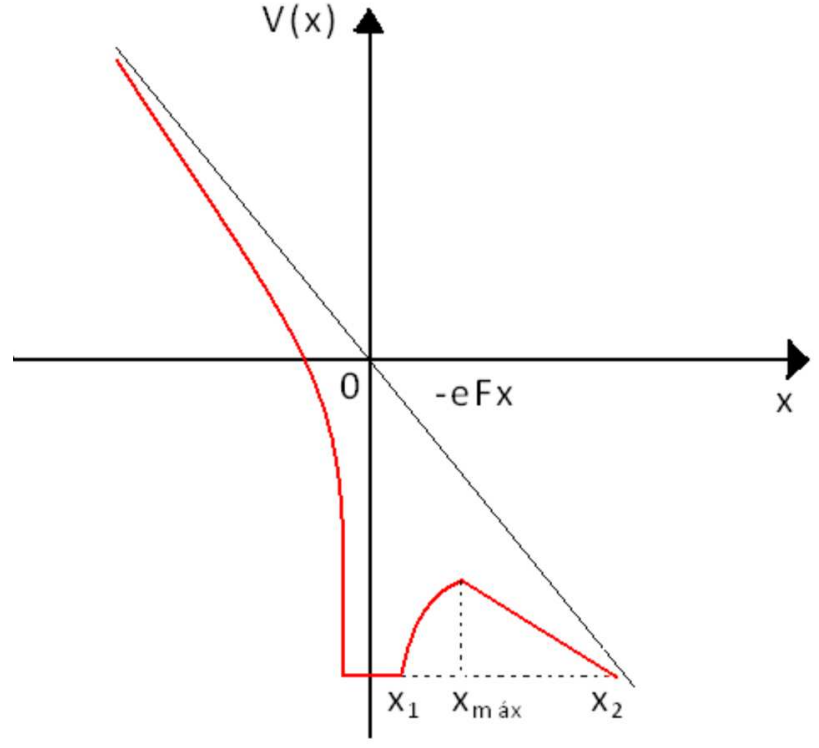

Figura 1 - Representação esquemática da energia potencial do modelo 1 . O potencial coulombiano atua até o ponto $x_{\max }$.

\subsection{Modelo 1}

No modelo 1 separaremos em regiões distintas as influências do campo elétrico e do campo coulombiano, conforme mostrado na Fig. 1. Próximo ao núcleo atômico a influência do potencial coulombiano predomina em comparação com a influência do campo elétrico no potencial total, ocorrendo o contrário a medida que nos afastamos do núcleo atômico. Desse modo, próximo ao núcleo vamos utilizar apenas o potencial coulombiano mas, a partir do máximo, a barreira decresce linearmente. Ficamos então com a seguinte expressão

$$
P=\exp \left[-2^{3 / 2}\left(\int_{B^{-1}}^{F^{-1 / 2}} \sqrt{B-1 / x} d x+\int_{F^{-1 / 2}}^{B / F} \sqrt{B-F x} d x\right)\right] .
$$

A primeira integral da equação acima é resolvida fazendo-se a substituição $y^{2}=B-1 / x$ e tem como resultado exato

$$
\int_{B^{-1}}^{F^{-1 / 2}} \sqrt{B-1 / x} d x=\frac{\sqrt{B-\sqrt{F}}}{\sqrt{F}}-\frac{1}{2 \sqrt{B}} \ln \left(\frac{\sqrt{B}+\sqrt{B-\sqrt{F}}}{\sqrt{B}-\sqrt{B-\sqrt{F}}}\right) .
$$

A segunda integral é trivial e tem como resultado

$$
\int_{F^{-1 / 2}}^{B / F} \sqrt{B-F x} d x=\frac{2}{3 F}(B-\sqrt{F})^{3 / 2} .
$$

Substituindo as Eqs. (14) e (15) na Eq. (13) e considerando $F<<B^{2}$, encontramos para a probabilidade de penetração da barreira

$$
P=\exp \left[-\frac{2(2 B)^{3 / 2}}{3 F}-\left(\frac{2}{B}\right)^{1 / 2} \ln \left(\frac{\sqrt{F}}{4 B}\right)+\left(\frac{2}{B}\right)^{1 / 2}\right]
$$




\section{2. $\quad$ Modelo 2}

Esse modelo é o análogo unidimensional do potencial real em três dimensões (Fig. 2). Substituindo a Eq. (8) na Eq. (6) a probabilidade de penetração da barreira fica agora

$$
P=\exp \left[-2^{3 / 2} \int_{x_{1}}^{x_{2}} \sqrt{B-1 /|x|-F x} d x\right]
$$

que é uma integral muito mais difícil de resolver comparadas com as integrais do modelo 1. Para resolvêla podemos reescrever o integrando que aparece na equação acima como

$$
L=\int_{x_{1}}^{x_{2}}\left(\frac{F}{x}\right)^{1 / 2} \sqrt{\left(x_{2}-x\right)\left(x-x_{1}\right)} d x
$$

onde

$$
x_{1}+x_{2}=\frac{B}{F}
$$

$$
x_{1} x_{2}=\frac{1}{F} .
$$

A integral (18) é tabelada (Ref. [10, p. 235]) e tem como resultado

$$
L=\frac{2 B^{1 / 2}}{3}\left[\frac{B}{F} J(p)-\frac{2}{B} K(p)\right]
$$

onde

$$
p^{2}=\frac{x_{2}-x_{1}}{x_{1}} .
$$

e $J(p)$ e $K(p)$ são integrais elipiticas completas que podem ser desenvolvidas em série [10]

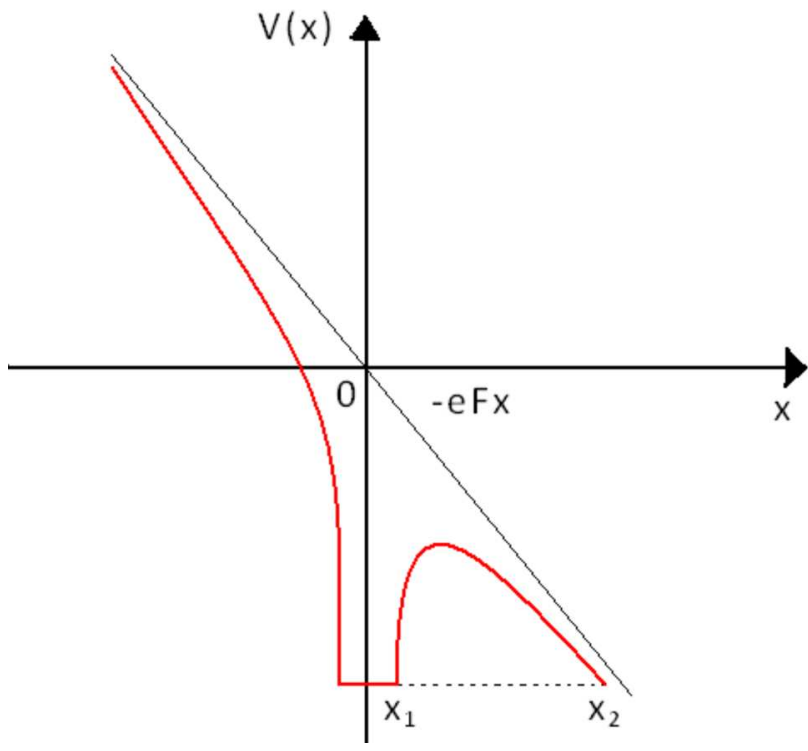

Figura 2 - Representação esquemática da energia potencial do modelo 2. O potencial coulombiano atua sobre todo o espaço.

$$
\begin{aligned}
& J\left(p^{\prime}\right)=1+\frac{1}{2}\left[\ln \frac{4}{p^{\prime}}-\frac{1}{2}\right] p^{2} \\
& K\left(p^{\prime}\right)=\ln \frac{4}{p^{\prime}}+\left[\ln \frac{4}{p^{\prime}}-1\right] \frac{p^{\prime}}{4}
\end{aligned}
$$

onde

$$
p^{\prime}=\left(\frac{x_{1}}{x_{2}}\right)^{1 / 2}=\frac{F^{1 / 2}}{B} .
$$

Substituindo as Eqs. (23) e (24) na Eq. (21) obtemos

$$
L=\frac{2 B^{3 / 2}}{3 F}-\frac{\ln \left(4 B F^{-1 / 2}\right)}{B^{1 / 2}}-\frac{1}{6 B^{1 / 2}}
$$

Utilizando a Eq. (26), a Eq. (17) fica

$$
P=\exp \left[-\frac{2(2 B)^{3 / 2}}{3 F}-\left(\frac{8}{B}\right)^{1 / 2} \ln \left(\frac{F^{1 / 2}}{4 B}\right)+\frac{2^{1 / 2}}{3 B^{1 / 2}}\right]
$$

Calculadas as probabilidades de penetração de barreira, podemos determinar a taxa de ionização.

\section{Expressões para a taxa de ionização}

Obtidas as probabilidades de penetração de barreira pelos modelos acima, Eq. (16) e Eq. (27), podemos agora obter a taxa de ionização do átomo de hidrogênio. Vamos considerar o elétron no estado fundamental. Nessa situação sua energia é deslocada pelo efeito do campo (efeito Stark) e vale $B=1 / 2+(9 / 4) F^{2}$. Substituindo esse valor nas Eqs. (16) e (27) e utilizando a Eq. (5) encontramos para a taxa de ionização segundo os modelos utilizados

$$
I_{1}=2 F^{-1} \exp \left(-\frac{2}{3 F}+2\right) \approx 14,7 F^{-1} \exp \left(-\frac{2}{3 F}\right) .
$$

$$
I_{2}=8 F^{-2} \exp \left(-\frac{2}{3 F}+\frac{2}{3}\right) \approx 15,6 F^{-2} \exp \left(-\frac{2}{3 F}\right) .
$$

Observamos das Eqs. (28) e (29) que a taxa de ionização é fortemente dependente do modelo utilizado, já que temos resultados bastante diferentes para mesmo valores de campo. Outro resultado notável é a forte dependência do campo elétrico já que pequenas alterações no valor do campo elétrico podem mudar a taxa de ionização por várias ordens de grandeza, conforme podemos observar dos valores da Tabela 1 . 
Tabela 1 - Valores numéricos para a taxa de ionização para diversos valores de campo. $I_{1}, I_{2}$ e $I_{L a n d a u}$ foram, respectivamente, calculadas pelas Eqs. (28), (29) e (30) e $I_{n u m}$ foi obtida por integração numérica.

\begin{tabular}{ccccc}
\hline$F$ (u.a.) & $I_{1}$ (u.a.) & $I_{2}$ (u.a.) & $I_{\text {Landau }}($ u.a. $)$ & $I_{n u m .}($ u.a. $)$ \\
\hline 0,01 & $1.65 \times 10^{-26}$ & $1.74 \times 10^{-24}$ & $4.48 \times 10^{-27}$ & $6.95 \times 10^{-24}$ \\
0,02 & $2.47 \times 10^{-12}$ & $1.30 \times 10^{-10}$ & $6.68 \times 10^{-13}$ & $5.29 \times 10^{-10}$ \\
0,03 & $1.10 \times 10^{-7}$ & $3.87 \times 10^{-6}$ & $2.98 \times 10^{-8}$ & $1.57 \times 10^{-5}$ \\
0,04 & $2.13 \times 10^{-5}$ & $5.62 \times 10^{-4}$ & $5.78 \times 10^{-6}$ & $2.28 \times 10^{-3}$ \\
\hline
\end{tabular}

Na Tabela 1 são mostrados os valores numéricos para a taxa de ionização em função do campo elétrico no intervalo $0,01<F<0,04$ (em u.a.). $I_{1}$ é a expressão obtida do modelo $1, I_{2}$ é a expressão obtida do modelo

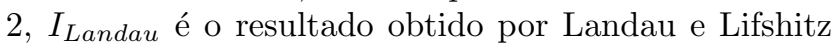
e $I_{\text {num. }}$. é o resultado obtido por integração numérica utilizando o potencial do modelo 2 . Vemos que $I_{n u m}$. e $I_{2}$ possuem a mesma ordem de grandeza (mais exatamente $I_{n u m} \approx 4 I_{2}$ ). Do mesmo modo, $I_{\text {Landau }}$ e $I_{1}$ possuem a mesma ordem de grandeza (mais exatamente $\left.I_{1} \approx 3,7 I_{\text {Landau }}\right)$

No limite que estamos considerando $\left(F<<B^{2}\right)$, o seguinte resultado, obtido por Landau e Lifshitz [11], é o aceito como o correto [4]

$$
I_{\text {Landau }}=4 F^{-1} \exp \left(-\frac{2}{3 F}\right) \text {. }
$$

A Eq. (30) é obtida resolvendo a equação de Schrödinger tridimensional em coordenadas parabólicas. Devemos então comparar os nossos resultados com a expressão de Landau e Lifshitz.

Vemos que o resultado obtido via modelo 1, Eq. (28), a menos de um fator numérico, depende do campo elétrico de forma idêntica ao resultado de Landau e Lifshitz $\left[F^{-1} \exp (-2 / 3 F)\right]$. Já o resultado obtido via modelo 2, Eq. (29), é bastante diferente. Outro aspecto importante a ressaltar é que Landau e Lifshitz resolvem a equação de Schrödinger tridimensional. Devemos, então, justificar o uso de modelos unidimensionais. Em primeiro lugar, os modelos unidimensionais são bastante simples, de modo que sua utilização é perfeitamente justificável de um ponto de vista pedagógico. Em segundo lugar, de um ponto de vista mais fundamental, o elétron emitido possui momento angular nulo (estamos considerando o estado fundamental) o que significa que, numa visão clássica, a trajetória do elétron é aproximadamente linear. Como terceira razão temos que, mesmo no caso tridimensional, a probabilidade de tunelamento é maior na região para ângulos $\theta$ próximos de zero. Assim a Eq. (2) basicamente depende de uma única variável.

\section{Conclusões}

Utilizando a aproximação WKB e potenciais unidimensionais, neste trabalho foi apresentado um método relativamente simples para calcular a taxa de iønização do átomo de hidrogênio quando submetido a um campo elétrico uniforme, o que possibilita ao estudante de graduação tratar de um importante fenômeno quântico (ionização por campo) sem a necessidade de resolver a equação de Schrödinger em coordenadas parabólicas. Deve-se observar que o fator $\exp (-2 / 3 F)$ é comum aos dois modelos mas o fator pré-exponencial é bastante diferente nos dois casos. O potencial coulombiano causa um fator pré-exponencial de $14,7 F^{-1}$ se ele é considerado apenas antes do máximo do potencial, e de $15,6 F^{-2}$ se considerado sobre toda a barreira, enquanto no resultado de Landau e Lifshitz esse fator vale $4 F^{-1}$. A integral a ser resolvida no modelo 2 , que é uma versão unidimensional do potencial real tridimensional, do tipo elíptica, é bastante difícil e para sua solução tem-se que fazer expansões em séries. Por essa razão, também utilizamos o modelo 1 que, além do mesmo conter todas as características físicas importantes do sistema, as integrais a serem resolvidas são simples e permitem soluções analíticas exatas. Não obstante sua simplicidade, o modelo 1 apresenta concordância com o resultado de Landau e Lifshitz muito melhor do que o modelo 2. Contudo, os resultados numéricos obtidos do potencial "real" estão muito mais próximos do resultado do modelo 2 .

\section{Apêndice}

Hartre em 1928 foi o primeiro a utilizar um sistema de unidades que ficaria conhecido como sistema de unidades atômicas, cuja utilização permite uma economia de fatores numéricos na realização de cálculos na escala atômica. Estas unidades surgem das combinações de três grandezas fundamentais, a saber, a carga do elétron $e$, a massa do elétron $m$ e a constante de Planck reduzida, $\hbar$, tembém denominada de constante de Dirac.

As principais grandezas físicas em unidades atômicas estão relacionadas a seguir, bem como seus valores numéricos em unidades familiares:

- Unidade de carga: A magnitude da carga do elétron (e) cujo valor é $4,8029 \times 10^{-10}$ esu no CGS ou $1,6021 \times 10^{-19} \mathrm{C}$ no SI.

- Unidade de massa: A massa do elétron $(m)$ cujo valor é $9,108 \times 10^{-28}$ g no CGS ou $9,108 \times$ $10^{-28} \mathrm{~kg}$ no SI. 
- Unidade de ação: A constante de Planck reduzida ( $\hbar$ ) cujo valor é $1,0544 \times 10^{-27} \mathrm{erg} \cdot \mathrm{s}$ no CGS ou $1,145 \times 10^{-34} \mathrm{~J} \times \mathrm{s}$ no SI.

- Unidade de comprimento: O raio da primeira órbita de Bohr $\left(a_{o}\right) a_{o}=\hbar^{2} /\left(m e^{2}\right)=5,29 \times$ $10^{-9} \mathrm{~cm}$ ou $0,529 \AA$ A.

- Unidade de velocidade: A velocidade do elétron na primeira órbita de $\operatorname{Bohr}\left(v_{o}\right) v_{o}=e^{2} / \hbar=$ $2,19 \times 10^{8} \mathrm{~cm} / \mathrm{s}$.

- Unidade de energia: Duas vezes a energia de ionização do átomo de hidrogênio $\left(e^{2} / a_{o}=4,36 \times\right.$ $10^{-11}$ erg ou $\left.27,2 \mathrm{eV}\right)$.

- Unidade de tempo: O tempo gasto para o elétron percorrer uma distância correspondente ao raio de Bohr com a velocidade do elétron na primeira órbita de Bohr $\left(a_{o} / v_{o}=\hbar^{3} /\left(m e^{4}\right)=2,42 \times\right.$ $\left.10^{-17} \mathrm{~s}\right)$.

- Unidade de momento: O momento do elétron na primeira órbita de Bohr $\left(p_{o}\right) p_{o}=m e^{2} / \hbar=$ $1,9 \times 10^{-19} \mathrm{~g} \mathrm{~cm} / \mathrm{s}$.

- Unidade de campo elétrico: $\left(e / a_{o}^{2}\right) e / a_{o}^{2}=5,14 \times$ $10^{9} \mathrm{~V} / \mathrm{cm}=51,4 \mathrm{~V} / \AA$.

\section{Referências}

[1] H. Moysés Nussenzveig, Curso de Fúsica Básica 4: Ótica, Relatividade, Física Quântica (Editora Edgar Blücher, 2002).

[2] R. Oppenheimer, Phys. Rev. 31, 67 (1928).

[3] Caio Mário Castro de Castilho, Revista Brasileira de Ensino de Física 25, 364 (2003).

[4] Y. Yamabe, A Tashibama and H.J. Silverstone, Phys. Rev. A 16, 877 (1977).

[5] Roger Haydock and David R. Kingham, J. Phys. B; Atom. Mol. Phys. 14, 385 (1981).

[6] Alexandre Marletta e Euclydes Marega Jr., Revista Brasileira de Ensino de Física 19, 106 (1997).

[7] A.V. Andrade-Neto and C.M. Castro de Castilho, J. Phys. B; Atom. Mol. Phys. 24, 2609 (1991).

[8] David Bohm, Quantum Theory (Prentice-Hall, New Jersey, 1960).

[9] David J. Griffiths, Introduction to Quantum Mechanics (Pearson Education, New Jersey, 2005).

[10] I.S. Gradshteyn and I.M. Ryzhik, Tables of Integrals, Series and Products (Academic, Nova York, 1965).

[11] L.D. Landau and E.M. Lifshitz, Quantum Mechanics (Pergamon, Oxford, 1965), §77. 